AF that is measurable, fits within the current NHS landscape and maximises clinicians and managers time effectively. We have educated over 100 clinicians in the use of the pathway to date.

We anticipate the this pathway will increase the detection of AF, increase in the number of people with $\mathrm{AF}$ treated with effective and appropriate AC, reduce the number of people with AF related stroke and increase support to clinicians, providers and commissioning organisations.

We are currently evaluating this pathway over a 24 month period at both general practice and network level using GRASP-AF, $\mathrm{CHA}_{2} \mathrm{DS}_{2}$-VASc and other KPIs within the pathway. We anticipate that this pathway will benefit all professional stakeholders involved in AF care but more importantly, improve outcomes for people with AF.

\section{IMPACT OF THE INTRODUCTION OF A STANDARDISED ICD PROGRAMMING PROTOCOL: REAL-WORLD DATA FROM A SINGLE CENTRE}

Nicholas Sunderland*, Amit Kaura, Anthony Li, Ravi Kamdar, Ed Petzer, Para Dhillon, Francis Murgatroyd, Paul Scott. King's College Hospital; *Presenting Author

\subsection{6/heartjnl-2016-309890.51}

Aims Randomised trials have shown that empiric ICD programming, using long detection times and high detection zones, reduces device therapy in ICD recipients. However, there is less data on its effectiveness in a "real-world" setting, especially secondary prevention patients. Our aim was to evaluate the introduction of a standardised programming protocol in a "real-world" setting of unselected ICD recipients.

Methods We analysed 270 consecutive ICD recipients implanted in a single centre - 135 implanted prior to protocol implementation (Physician-Led group) and 135 after (Standardised group). The protocol included long arrhythmia detection times (30/40 or equivalent) and high rate detection zones (primary prevention lower treatment zone $200 \mathrm{bpm}$ ). Programming in the Physician-Led group was at the discretion of the implanter. The primary endpoint was time-to-any therapy (ATP or shocks). Secondary endpoints were time-to-inappropriate therapy and time-to-appropriate therapy. The safety endpoints were syncopal episodes, hospital admissions, and death.

Results At 12 months follow-up, 47 patients had received any ICD therapy (Physician-Led group, $\mathrm{n}=31$ vs. Standardised group, $\mathrm{n}=16$ ). There was a $47 \%$ risk reduction in any device therapy $(\mathrm{p}=0.04)$ and an $86 \%$ risk reduction in inappropriate therapy $(p=0.009)$ in the Standardised compared to the Physician-led group. Results were consistent across primary and secondary prevention patients. There were no significant differences in the rates of syncope, hospitalization and death.

Conclusions In unselected patients in a "real-world" setting introduction of a standardised programming protocol, using long detection times and high detection zones, significantly reduces the burden of ICD therapy without an increase in adverse outcomes.

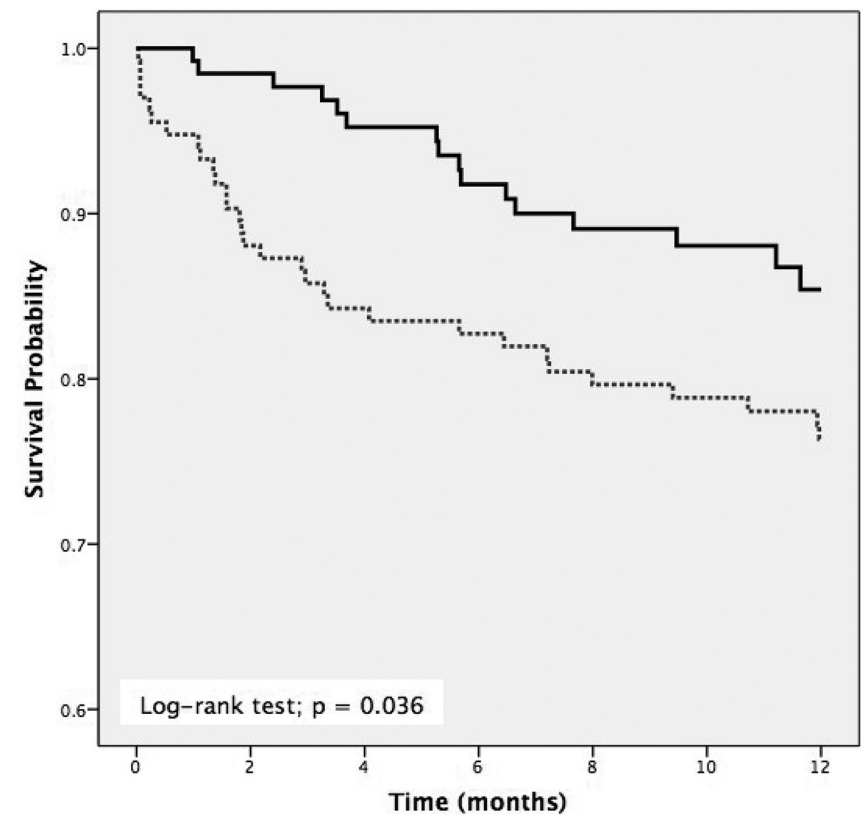

Abstract 51 Figure 1 Kaplan-Meier curves for survival from anytherapy. Physician-Led (dotted) vs. Standardised (black)

\section{IMMEDIATE MANAGEMENT FOLLOWING CARDIAC IMPLANTABLE ELECTRONIC DEVICE PROCEDURES; WIDE VARIATION IN PRACTICE FROM A UK SURVEY}

Robert Bowers*, Alison Parish, Richard Balasubramaniam, John Paisey, Mark Sopher. ${ }^{1}$ Royal Bournemouth Hospital; *Presenting Author

\subsection{6/heartjnl-2016-309890.52}

Introduction Following the implantation of a CIED a number of checks are made to document device function and exclude procedure complications. Traditionally these have been performed the day after the procedure, mandating an overnight admission. Recently, both the need for these investigations and

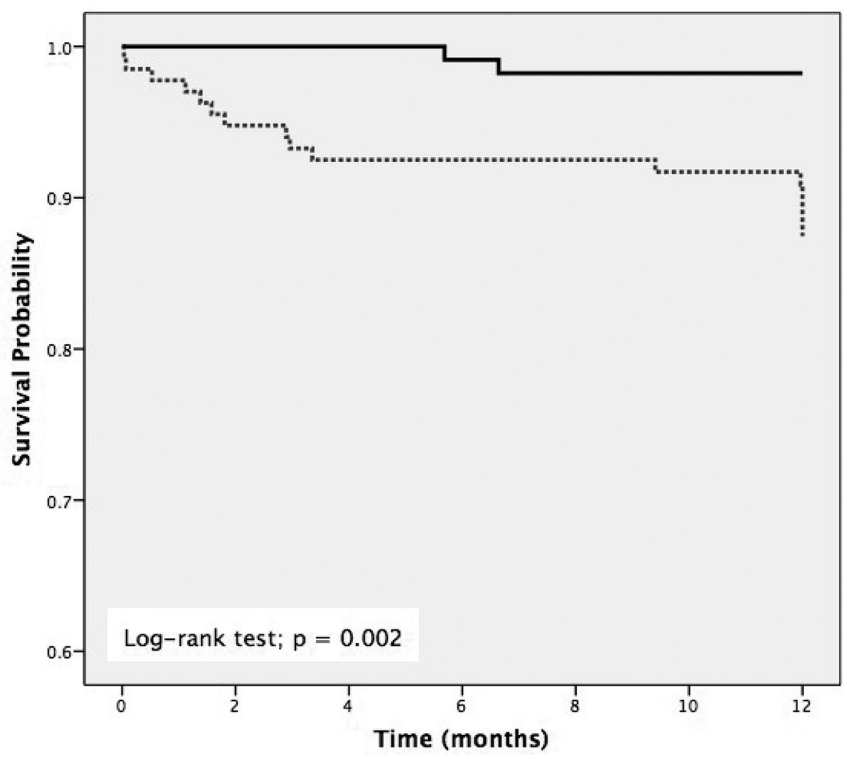

Abstract 51 Figure 2 Kaplan-Meier curves for survival from inappropriate therapy. Physician-led (dotted) vs. Standardised (black) 